J.M. Pérez, J.Ma. Rincón, M. Romero, Study of mullite formation in porcelain stoneware applying isoconversional and IKP methods. Ceramics International, Volume 36, Issue 8, December 2010, Pages 2329-2335; doi:10.1016/j.ceramint.2010.08.001.

\title{
Study of mullite formation in porcelain stoneware applying isoconversional and IKP methods
}

\author{
J.M. Pérez, J.Ma. Rincón, M. Romero
}

Group of Glassy and Ceramic Materials, Instituto de Ciencias de la Construcción Eduardo Torroja, CSIC, C/Serrano Galvache 4, 28033 Madrid, Spain

\begin{abstract}
The growth process of mullite in a porcelain stoneware body has been studied under isoconversional, isokinetic relationship and invariant kinetic parameters. Activation energy for mullite crystallisation of over $589-628 \mathrm{~kJ} \mathrm{~mol}^{-1}$ and a $\mathrm{Ln} A$ over $50-59 \mathrm{~min}^{-1}$ was obtained. The model was Johnson-Melh-Avrami with $n=1.5$. The model chosen implies quick nucleation and subsequent one or three-dimensional growth. Isoconversional methods show an independent activation energy variation in mid range conversion degrees. Lower and higher conversion degrees show different reactions in mullite formation. Results obtained with the methods employed here are in agreement with a previous paper where the Kissinger non-isothermal method and Ligero et al.approximation were applied.
\end{abstract}

\section{Keywords}

D. Mullite; Compensation effect; DTA; Porcelain stoneware

\section{Introduction}

Within the wide range of building ceramic products, it highlights the great development of porcelain stoneware tile, which was developed in the 1980s as a very compact vitrified product with high technical performance. Porcelain stoneware typically has a triaxial composition comprised of kaolinitic clay, flux and filler. Firing bodies containing these three components result in a grain and bond microstructure consisting of coarse quartz grains held together by a finer bond or matrix consisting of mullite crystals and a glassy phase [1]. Because of kinetic limitations and complexities of the microstructure and phase development [2], porcelain materials represent some of the most complicated of all ceramic materials. 
J.M. Pérez, J.Ma. Rincón, M. Romero, Study of mullite formation in porcelain stoneware applying isoconversional and IKP methods. Ceramics International, Volume 36, Issue 8, December 2010, Pages 2329-2335; doi:10.1016/j.ceramint.2010.08.001.

Mullite crystals are derived from the solid-state decomposition of the clay component, and are endowed with excellent mechanical, creep, thermal and chemical properties [3]. It has been probed that mullite content affects the mechanical properties of porcelain stoneware. Thus, it was reported that the tile strength increases with increasing mullite content [4],[5] and [6]. Consequently, knowledge of the kinetic of mullite formation on firing is of great importance for both enhancing the technological properties of porcelain stoneware tiles and optimising their fast-firing schedule.

Non-isothermal methods are most commonly used to perform the kinetic analysis of solidstate reactions. There are many ways to study the reaction under non-isothermal conditions. Isoconversional methods have generally been used to determine the activation energy as a function of the reacted fraction without any previous assumption on the kinetic model fitted by the reaction. Isokinetic methods are those in which the transformation mechanism is assumed to be the same throughout the temperature/time range of interest. These methods also suppose that the kinetic parameters are constant with respect to time and temperature.

In the literature, the application of the Isokinetic Relationship (IKR) and Invariant Kinetic Parameters (IKP) methods can be found for studying the decomposition of aromatic azomonoethers [7], polymer curing [8], polymerisation of poly(ester amide) potassium salt[9], nickel oxide reduction [10], and the crystallisation of silica-soda-lead glass [11]. However, to the author's knowledge, these methods have not been previously applied to the study of mullite crystallisation in porcelain stoneware.

The aim of the present work is to apply the previous methods (isoconversional; integral and differential using a single-heating rate; and the IKR and IKP methods) to obtain the kinetics parameters of mullite formation. In order to get the $n$-order of the reaction and check the previous results, the Pérez-Maqueda et al. [12] criterion was used.

\section{Experimental}

\subsection{Materials and methods}

A standard porcelain stoneware body for tile production was prepared by mixing $50 \%$ kaolinitic clay (EuroArce), 40\% feldspar (Rio Pirón) and 10\% quartz sand, as previously reported [3]. 
J.M. Pérez, J.Ma. Rincón, M. Romero, Study of mullite formation in porcelain stoneware applying isoconversional and IKP methods. Ceramics International, Volume 36, Issue 8, December 2010, Pages 2329-2335; doi:10.1016/j.ceramint.2010.08.001.

Differential thermal analysis (DTA) was performed in a SETARAM Labsys Thermal Analyzer. The samples were tested in platinum crucibles with calcinated $\mathrm{Al}_{2} \mathrm{O}_{3}$ as reference material, from room temperature to $1523 \mathrm{~K}$, at heating rates of $10,20,30,40$ and $50 \mathrm{~K} \mathrm{~min}^{-1}$. The obtained data were applied to isoconversional Flynn-Wall-Ozawa [13] and [14] and Kissinger-Akahira-Sunose methods [15]; integral and differential one single-heating rate; and the isokinetic parameter method.

\subsection{Kinetic methods}

\subsubsection{Isoconversional methods}

The isoconversional methods employed in this work are based on dynamic DTA analysis. The equation for the reaction rate employed to study the degree of crystallisation can be expressed, in general, as follows:

$\frac{d x}{d t}=k(T) \cdot f(x)$

where $x$ is the extent of the reaction. Considering that Eq. (1) is valid for dynamic curing and that $d x / d t=\beta(d x / d T)$, where $\beta$ is the heating rate $\left(\mathrm{K} \mathrm{min}^{-1}\right)$ and $k(T)$ is the rate constant expressed by the temperature-dependent Arrhenius equation, Eq. (1) can be written as:

$\beta \cdot \frac{d x}{d T}=A \cdot e^{(-\mathrm{Ea} / R \cdot T)} \cdot f(x)$

where $T$ is the temperature, $A$ is the pre-exponential factor, Ea is the activation energy and $R$ is the gas constant. Therefore, the equation can be expressed in its integral form as:

$g(x)=\int_{0}^{x} \frac{d x}{f(x)}=\frac{A}{\beta} \int_{0}^{T} e^{(-E a / R \cdot T)} d T$

It was assumed that the p-Doyle function, $P(E / R T)$, can be expressed according to the $20-60$ $\mathrm{Ea} / R T$ range [16], and hence we obtain the following:

$\operatorname{Ln} \beta=C t e-1.052 \cdot \frac{\mathrm{Ea}}{R \cdot T}$ 
J.M. Pérez, J.Ma. Rincón, M. Romero, Study of mullite formation in porcelain stoneware applying isoconversional and IKP methods. Ceramics International, Volume 36, Issue 8, December 2010, Pages 2329-2335; doi:10.1016/j.ceramint.2010.08.001.

Expression (4) is known as the Flynn-Wall-Ozawa (FWO) method and can be applied to different conversion degrees. Thus, according to Eq. (4), the activation energy and the constant $C t e$ can be calculated using the slope and the ordinate values expressed by the linear relationship between $\operatorname{Ln} \beta$ and 1/T.

A similar expression is the Kissinger-Akahira-Sunose (KAS) model-free method that can be obtained by applying the Murray and White approximation [17]:

$\operatorname{Ln}\left(\frac{\beta}{T^{2}}\right)=\operatorname{Ln}\left(\frac{R \cdot A}{\mathrm{Ea} \cdot g(x)}\right)-\frac{\mathrm{Ea}}{R} \cdot \frac{1}{T}$

In this case, the method does not require knowledge of the conversion-dependent function $(f(x)$ or $g(x)$ ), and only assumes that the process follows the same mechanism of reaction for a given conversion degree, regardless of the crystallisation temperature.

\subsubsection{Single $\mathrm{x}=\mathrm{x}(\mathrm{T})$ curve methods}

These methods obtain the kinetic parameters with a single-heating rate. In this work, we employ the following:

- the integral method known as the Coats-Redfern (C-R) method [18]:

$\operatorname{Ln}\left[\frac{g(x)}{T^{2}}\right]=\operatorname{Ln}\left[\frac{A \cdot R}{\beta \cdot \mathrm{Ea}}\right]-\frac{\mathrm{Ea}}{R \cdot T}$

- the differential method (D) based on Eq. (4):

$\operatorname{Ln}\left[\frac{d x / d t}{f(x)}\right]=\operatorname{Ln} A-\frac{\mathrm{Ea}}{R \cdot T}$

For a given model (Table 1) and heating rate, the linear plot of the left-hand sides $v s$. 1/Tallowed us to obtain the Ea and $A$ from the slope and the intercept. 
J.M. Pérez, J.Ma. Rincón, M. Romero, Study of mullite formation in porcelain stoneware applying isoconversional and IKP methods. Ceramics International, Volume 36, Issue 8, December 2010, Pages 2329-2335; doi:10.1016/j.ceramint.2010.08.001.

Table 1. Expressions of $f(x)$ and $g(x)$ functions.

\begin{tabular}{cccc} 
Mechanism & Symbol & $\mathrm{f}(\mathrm{x})$ & $\mathrm{g}(\mathrm{x})=_{0}^{a} \frac{1}{f(x)} \mathrm{dx}$ \\
\hline Avrami equation $\mathrm{n}=1.5$ & $\mathrm{~A}_{3 / 2}$ & $(3 / 2)(1-\mathrm{x})[-\operatorname{Ln}(1-\mathrm{x})]^{1 / 3}$ & {$[-\operatorname{Ln}(1-\mathrm{x})]^{2 / 3}$}
\end{tabular}

Two-dimensional nucleation

(Avrami 1 equation)

Two-dimensional growth of nuclei (Avrami 2 equation)

Three-dimensional growth of nuclei (Avrami 3 equation)

Zero-order mechanism

Phase-boundary-controlled

reaction (contracting

$$
\text { cylinder) }
$$

Phase-boundary-controlled reaction (contracting sphere)

One-dimensional diffusion

Two-dimensional diffusion

Three-dimensional diffusion

(Jander equation)

Three-dimensional diffusion

(Ginsting-Brounshtein equation)

First order reaction

Second order reaction

Third order reaction
$\mathrm{A}_{2}$
$2 \cdot(1-x) \cdot[-\operatorname{Ln}(1-x)]^{1 / 2}$
$[-\operatorname{Ln}(1-x)]^{1 / 2}$
$\mathrm{A}_{3}$
$3 \cdot(1-x) \cdot[-\operatorname{Ln}(1-x)]^{2 / 3}$
$[-\operatorname{Ln}(1-x)]^{1 / 3}$

$\mathrm{A}_{4}$

$4 \cdot(1-x) \cdot[-\operatorname{Ln}(1-x)]^{3 / 4}$

$[-\operatorname{Ln}(1-x)]^{1 / 4}$

$\mathrm{R}_{1}$

1 $\mathrm{X}$

$\mathrm{R}_{2}$

$2 \cdot(1-x)^{1 / 2}$

$\left[1-(1-x)^{1 / 2}\right]$

$\mathrm{R}_{3}$

$3 \cdot(1-x)^{2 / 3}$

$\left[1-(1-x)^{1 / 3}\right]$

$\mathrm{D}_{1}$

$1 / 2 \mathrm{x}$

$x^{2}$

$\mathrm{D}_{2}$

$$
\frac{1}{-\operatorname{Ln}(1-x)}
$$$$
(1-x) \cdot \operatorname{Ln}(1-x)+x
$$

$\mathrm{D}_{3} \quad \frac{3 \cdot(1-x)^{2 / 3}}{2 \cdot\left[1-(1-x)^{1 / 3}\right]}$

$$
\left[1-1-x^{1}{ }^{3}\right]^{2}
$$
$\mathrm{D}_{4}$
$\frac{3}{2 \cdot\left[1-x^{13}-1\right]}$
$1-\frac{2}{3} \mathrm{x}-(1-x)^{2 / 3}$

$\mathrm{F}_{1}$

$(1-x)$

$-\operatorname{Ln}(1-x)$

$\mathrm{F}_{2}$

$(1-x)^{2}$

$\frac{1}{(1-x)}$

$\mathrm{F}_{3}$

$(1-x)^{3}$ 
J.M. Pérez, J.Ma. Rincón, M. Romero, Study of mullite formation in porcelain stoneware applying isoconversional and IKP methods. Ceramics International, Volume 36, Issue 8, December 2010, Pages 2329-2335; doi:10.1016/j.ceramint.2010.08.001.

\subsubsection{Compensation effect}

The activation energy and the pre-exponential factor may be joined together by the so-called compensation effect or isokinetic relationship (IKR) through the following equation[19] and [20]:

$\operatorname{Ln} A_{x}=\alpha^{*}+\beta^{*} E_{x}$

where $\alpha^{*}$ and $\beta^{*}$ are constants and the $x$ subscripts refer to a factor producing a change in the Arrhenius parameters (conversion, heating rate and model). The intercept $\alpha^{*}=\operatorname{Ln} k_{\text {iso }}$ is related to the isokinetic rate constant $\left(k_{\text {iso }}\right)$. The slope $\beta^{*}=1 / R T_{\text {iso }}$ is related to the isokinetic temperature $\left(T_{\text {iso }}\right)$. The appearance of the IKR shows that only one mechanism is present, whereas the existence of parameters that do not agree with the IKR implies that there are multiple reaction mechanisms [20].

According to the results reported in [19], we may select the model whose IKR in relation to the conversion had the best linear correlation and in which the associated $T_{\text {iso }}$ value was near the experimental temperature range.

\subsubsection{Invariant kinetic parameters method (IKP)}

The IKP method [21] and [22] is based on the observation that the experimental curve $x(T)$ could be correctly described by several conversion functions. Using the apparent compensatory effect that exists when the model changes, for each heating rate $\left(\beta_{v}, v=1,2,3, \ldots\right)$ the compensation parameters $\alpha^{*}$ and $\beta^{*}$ are determined according to Eq. (8). A set of conversion functions, $f j, j=1,2,3, \ldots$ is also considered ( Table 1 ).

For each heating rate, $\beta_{v}$, the pairs $\left(\mathrm{A}_{\mathrm{vj}}, \mathrm{E}_{\mathrm{vj}}\right)$ characteristic of each conversion are determined using an integral or differential method. In this work, the integral method suggested by C-R (Eq. (6)), and the differential method based on Eq. (7) are used.

A plot of each of these equations should yield a straight line from which the intercept $(\operatorname{Ln}(A R / \beta \mathrm{Ea})$ or $\operatorname{Ln} A$, respectively) and slope $(-\mathrm{Ea} / R)$ can be obtained.

Using the relation of the apparent compensation effect, the compensation parameters $\alpha_{v}^{*}$

and $\beta_{v}^{*}$ are determined for each heating rate. The point of intersection of the straight lines 
J.M. Pérez, J.Ma. Rincón, M. Romero, Study of mullite formation in porcelain stoneware applying isoconversional and IKP methods. Ceramics International, Volume 36, Issue 8, December 2010, Pages 2329-2335; doi:10.1016/j.ceramint.2010.08.001.

corresponds to the true values of $A$ and $E$. These were called by Lesnikovich and Levchik[21] and [22] as invariant parameters $\left(A_{\text {inv }}, E a_{\text {inv }}\right)$, as they are independent of the conversion, the model and the heating rate. The evaluation of the invariant activation parameters is performed using the supercorrelation:

$\alpha_{v}^{*}=\operatorname{Ln} A_{\text {inv }}-\beta_{v}^{*} \mathrm{Ea}_{\text {inv }}$

The straight line $\alpha_{v v s}^{*} . \beta_{v}^{*}$, allows us to determine the invariant kinetic parameters ( $\left(\mathrm{Ea}_{\text {inv }}\right.$ and $\left.A_{\text {inv }}\right)$ from its slope and intercept.

It was pointed out that the values of the invariant conversion function are proportional to their true values [23] and [24]. Therefore, the IKP method aims to determine the invariant parameters independently of the kinetic model, comparing them to those obtained using other methods. This also allows us to decide which kinetic model best describes the process.

\subsubsection{Pérez-Maqueda et al. criterion}

In order to obtain the appropriate conversion function, one can discriminate by applying the Pérez-Maqueda et al. criterion. They have drawn the lines $\operatorname{Ln}[\beta(d x / d T) / f(x)] v s .1 / T$ for a set of conversion functions and for all heating rates. For a given function of conversion, one of the following curve families resulted: (a) non-linear curves; (b) linear curves, parallels, but with the intercept depending on the heating rate; and (c) one line for all the heating rates. The real conversion function corresponds to case (c). In the present paper, according to Eq. (5) there should be a straight line $\left(\operatorname{Ln}\left[\beta g(x) / T^{2}\right] v s .1 / T\right)$ for all heating rates. This will be also used for discrimination of the conversion function [7] and [11].

\section{Results and discussion}

In a previous work, the kinetic of mullite formation was obtained using non-isothermal DTA experiments [3]. In the present paper, employing the same experimental results, the Ea and Ln $A$ under several kinetic methods are obtained. The Kissinger and Ligero et al. [25]methods were formerly employed. The present methods are isoconversional (KAS and FWO). IKR is employed under $f(x)$ and heating rate variations. The IKP method is employed with $\mathrm{C}-\mathrm{R}$ and FR as integral and differential methods, respectively. Once $\mathrm{Ea}_{\text {inv }}$ and $\mathrm{Ln} A_{\text {inv }}$ were found, the Pérez- 
J.M. Pérez, J.Ma. Rincón, M. Romero, Study of mullite formation in porcelain stoneware applying isoconversional and IKP methods. Ceramics International, Volume 36, Issue 8, December 2010, Pages 2329-2335; doi:10.1016/j.ceramint.2010.08.001.

Maqueda et al. criterion was used to find the most suitable method and to check the results obtained.

\subsection{Isoconversional methods}

Fig. 1 shows the variation of activation energy with crystallisation degree. As can be seen, FWO and KAS give similar results in the whole range. Differences between the models come from different approximations of the temperature integral [11].

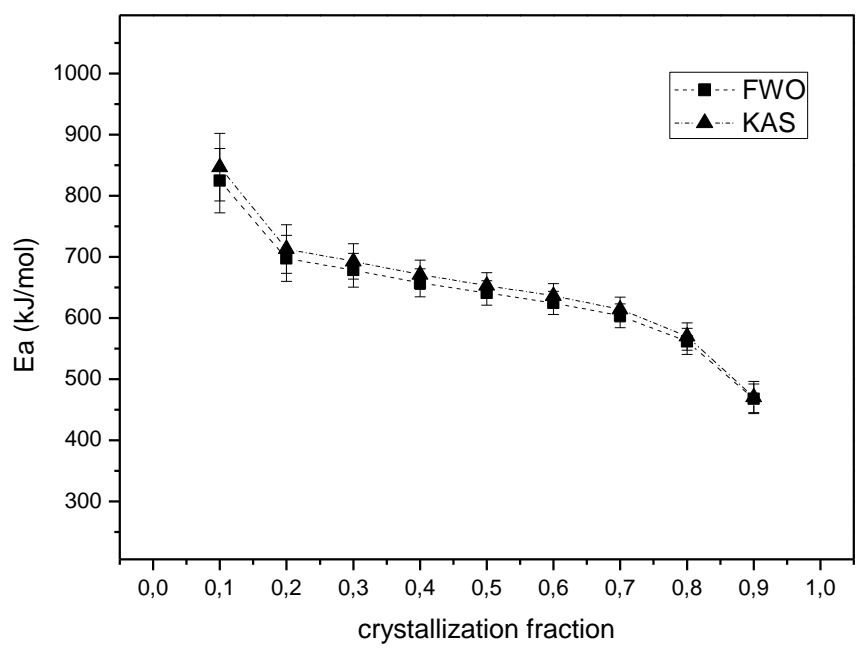

Fig. 1. Activation energy obtained by applying KAS and FWO isoconversional methods.

Taking into account this figure, lower and higher crystallisation degrees have proportionate activation energies with higher standard error than medium degrees (all the data have standard errors lower than 10\%). The noise may be serious when $x$ changes slowly, e.g., at the beginning and at the end of the reaction [26] and [27]. The kinetic results can be affected by inherent errors arising from $d x / d t$ values.

The shape of the activation energies among the models employed is very similar. One may distinguish three different variations in activation energy. At low and high degrees, the Ea varies with crystallisation degree, whereas the main part of the reaction shows that Ea does not significantly change. In the first part (lower crystallisation degree), changes of activation energy 
J.M. Pérez, J.Ma. Rincón, M. Romero, Study of mullite formation in porcelain stoneware applying isoconversional and IKP methods. Ceramics International, Volume 36, Issue 8, December 2010, Pages 2329-2335; doi:10.1016/j.ceramint.2010.08.001.

up to $20 \%$ may be caused by the transformation of metakaolinite into spinel or $\gamma$-alumina and amorphous silica, under either of these reactions [28],

$\mathrm{Al}_{2} \mathrm{O}_{3} \cdot 2 \mathrm{SiO}_{2}$ (metakaolinite) $\leftrightarrow \mathrm{SiAl}_{2} \mathrm{O}_{4}$ (spinel) $+\mathrm{SiO}_{2}$ (amorphous)

or

$\mathrm{Al}_{2} \mathrm{O}_{3} \cdot 2 \mathrm{SiO}_{2}$ (metakaolinite) $\leftrightarrow \mathrm{Al}_{2} \mathrm{O}_{3}\left(\gamma\right.$-alumina) $+2 \mathrm{SiO}_{2}$ (amorphous)

together with mullite formation [28] following these reactions,

$\mathrm{SiAl}_{2} \mathrm{O}_{4}$ (spinel) $+\mathrm{SiO}_{2}$ (amorphous) $\leftrightarrow 1 / 3\left(3 \mathrm{Al}_{2} \mathrm{O}_{3} \cdot 2 \mathrm{SiO}_{2}\right)($ mullite $)+4 / 3\left(\mathrm{SiO}_{2}\right)$

(amorphous)

or

$\mathrm{Al}_{2} \mathrm{O}_{3}\left(\gamma\right.$-alumina) $+2 \mathrm{SiO}_{2}$ (amorphous) $\leftrightarrow 1 / 3\left(3 \mathrm{Al}_{2} \mathrm{O}_{3} \cdot 2 \mathrm{SiO}_{2}\right)($ mullite $)+4 / 3 \mathrm{SiO}_{2}$ (amorphous)

In the middle zone, from 20 to $70 \%$, activation energy remains constant. This means that only one mechanism governs the reaction. When the activation energy remains constant, the mean values are $E_{\mathrm{FWO}}=650.2 \pm 34.5 \mathrm{~kJ} \mathrm{~mol}^{-1}$ and $E_{\mathrm{KAS}}=663.0 \pm 36.5 \mathrm{~kJ} \mathrm{~mol}^{-1}$. The FWO and KAS are slightly higher than those obtained by applying the Kissinger and Ligero methods [3], but they are in range with previous results.

Finally, the concave decrease of $E$ on $x$ may correspond to changing the mechanism from kinetic to diffusion [13].

\subsection{Single $\alpha=\alpha(T)$ curve methods}

The $\mathrm{C}-\mathrm{R}$ and differential methods have been employed to determine the kinetic model within the range where the crystallisation degree is constant. Table 2 listed the results obtained for 10, 20, 30, 40 and $50 \mathrm{~K} \mathrm{~min}^{-1}$ for different applied methods: (a) integral method (C-R method, Eq. (6)) and (b) differential method (Eq. (7)). 
J.M. Pérez, J.Ma. Rincón, M. Romero, Study of mullite formation in porcelain stoneware applying isoconversional and IKP methods. Ceramics International, Volume 36, Issue 8, December 2010, Pages 2329-2335; doi:10.1016/j.ceramint.2010.08.001.

Table 2. Integral and differential parameters determined using $\mathrm{C}-\mathrm{R}$ and the Friedman method.

Model $\underline{\beta=10 \mathrm{~K} \mathrm{~min}^{-1}} \quad \beta=20 \mathrm{~K} \mathrm{~min}^{-1} \quad \beta=30 \mathrm{~K} \mathrm{~min}^{-1}$ $\beta=40 \mathrm{~K} \mathrm{~min}^{-1}$ $\beta=50 \mathrm{~K}_{\min ^{-1}}$

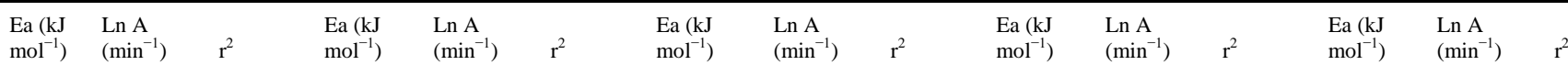

(a) Integral

\begin{tabular}{|c|c|c|}
\hline $\mathrm{A}_{3 / 2}$ & $\begin{array}{l}637.5 \pm \\
71.6\end{array}$ & $\begin{array}{l}57.75 \pm \\
18.26\end{array}$ \\
\hline $\mathrm{A}_{2}$ & $\begin{array}{l}378.8 \pm \\
53.7\end{array}$ & $\begin{array}{l}42.11 \pm \\
16.25\end{array}$ \\
\hline $\mathrm{A}_{3}$ & $\begin{array}{l}342.6 \pm \\
35.8\end{array}$ & $\begin{array}{l}26.90 \pm \\
14.12\end{array}$ \\
\hline $\mathrm{A}_{4}$ & $\begin{array}{l}251.8 \pm \\
26.8\end{array}$ & $\begin{array}{l}19.03 \pm \\
12.97\end{array}$ \\
\hline $\mathrm{R}_{1}$ & $\begin{array}{l}699.9 \pm \\
83.2\end{array}$ & $\begin{array}{l}64.34 \pm \\
19.53\end{array}$ \\
\hline $\mathrm{R}_{2}$ & $\begin{array}{l}606.1 \pm \\
94.2\end{array}$ & $\begin{array}{l}74.62 \pm \\
20.72\end{array}$ \\
\hline $\mathrm{R}_{3}$ & $\begin{array}{l}870.7 \pm \\
98.4\end{array}$ & $\begin{array}{l}78.61 \pm \\
21.16\end{array}$ \\
\hline $\mathrm{D}_{1}$ & $\begin{array}{l}1171.7 \\
\pm 166.3\end{array}$ & $\begin{array}{l}131.81 \pm \\
28.24\end{array}$ \\
\hline $\mathrm{D}_{2}$ & $\begin{array}{l}1168.7 \\
\pm 180.2\end{array}$ & $\begin{array}{l}145.04 \pm \\
29.65\end{array}$ \\
\hline $\mathrm{D}_{3}$ & $\begin{array}{l}1762.2 \\
\pm 196.7\end{array}$ & $\begin{array}{l}160.32 \pm \\
31.34\end{array}$ \\
\hline $\mathrm{D}_{4}$ & $\begin{array}{l}1639.3 \\
\pm 185.6\end{array}$ & $\begin{array}{l}149.30 \pm \\
30.21\end{array}$ \\
\hline $\mathrm{F}_{1}$ & $\begin{array}{l}750.8 \pm \\
107.3\end{array}$ & $\begin{array}{l}88.14 \pm \\
22.12\end{array}$ \\
\hline $\mathrm{F}_{2}$ & $\begin{array}{l}1297.0 \\
\pm 140.5\end{array}$ & $\begin{array}{l}118.43 \pm \\
25.58\end{array}$ \\
\hline $\mathrm{F}_{3}$ & $\begin{array}{l}1688.0 \\
\pm 182.9\end{array}$ & $\begin{array}{l}154.08 \pm \\
29.93\end{array}$ \\
\hline
\end{tabular}

$\begin{array}{llllll} & 669.4 \pm & 64.68 \pm & & 630.7 \pm & 59.85 \pm \\ 0.9085 & 9.7 & 10.97 & 0.9954 & 31.6 & 14.64 \\ & 514.7 \pm & 47.75 \pm & & 462.7 \pm & 43.68 \pm \\ 0.9049 & 7.2 & 10.46 & 0.9953 & 41.8 & 15.90 \\ & 336.1 \pm & 30.66 \pm & & 301.4 \pm & 28.04 \pm \\ 0.8974 & 4.8 & 9.82 & 0.9951 & 27.9 & 14.17 \\ & 246.9 \pm & 22.02 \pm & & 220.8 \pm & 20.12 \pm \\ 0.8895 & 3.6 & 9.42 & 0.9949 & 20.9 & 13.22 \\ & 803.1 \pm & 72.18 \pm & & 723.3 \pm & 68.31 \pm \\ 0.8963 & 25.4 & 13.44 & 0.9773 & 65.1 & 18.55 \\ & 920.8 \pm & 83.93 \pm & & 829.5 \pm & 77.98 \pm \\ 0.9057 & 21.6 & 12.92 & 0.9878 & 73.8 & 19.51 \\ & 962.7 \pm & 88.04 \pm & & 867.3 \pm & 81.25 \pm \\ 0.9081 & 20.0 & 12.70 & 0.9905 & 76.9 & 19.85 \\ & 1627.2 \pm & 146.67 \pm & & 1467.6 \pm & 138.84 \pm \\ 0.9012 & 45.6 & 15.96 & 0.9803 & 130.1 & 25.43 \\ & 1776.9 \pm & 161.70 \pm & & 1602.7 \pm & 151.24 \pm \\ 0.9069 & 46.5 & 16.06 & 0.9847 & 141.2 & 26.57 \\ & 1946.2 \pm & 178.40 \pm & & 1755.6 \pm & 164.53 \pm \\ 0.9120 & 40.1 & 15.30 & 0.9907 & 153.9 & 27.86 \\ & 1833.2 \pm & 166.23 \pm & & 1653.5 \pm & 154.65 \pm \\ 0.9088 & 44.3 & 15.81 & 0.9870 & 145.4 & 27.00 \\ & 1050.2 \pm & 98.46 \pm & & 946.3 \pm & 90.03 \pm \\ 0.9120 & 14.5 & 11.84 & 0.9955 & 83.6 & 20.56 \\ & 1344.3 \pm & 131.82 \pm & & 1211.9 \pm & 115.79 \pm \\ 0.9168 & 8.1 & 10.65 & 0.9993 & 106.2 & 22.96 \\ & 1684.0 \pm & 171.14 \pm & & 1518.5 \pm & 145.47 \pm \\ 0.9151 & 23.6 & 13.20 & 0.9963 & 133.1 & 25.74 \\ & & & & & \end{array}$

$\begin{array}{lll} & 586.1 \pm & 55.55 \pm \\ 0.9637 & 15.4 & 12.66 \\ & 444.9 \pm & 42.04 \pm \\ 0.9170 & 19.5 & 13.30 \\ & 289.6 \pm & 27.02 \pm \\ 0.9135 & 13.0 & 12.28 \\ & 211.9 \pm & 19.42 \pm \\ 0.9096 & 9.8 & 11.68 \\ & 696.0 \pm & 65.65 \pm \\ 0.9177 & 31.6 & 14.92 \\ & 798.4 \pm & 74.90 \pm \\ 0.9193 & 35.0 & 15.35 \\ & 834.8 \pm & 78.03 \pm \\ 0.9197 & 36.3 & 15.51 \\ & 1413.2 \pm & 133.27 \pm \\ 0.9198 & 63.1 & 18.59 \\ & 1543.4 \pm & 145.15 \pm \\ 0.9207 & 67.5 & 19.08 \\ & 1690.7 \pm & 157.85 \pm \\ 0.9215 & 72.6 & 19.64 \\ & 1592.4 \pm & 148.37 \pm \\ 0.9210 & 69.2 & 19.26 \\ & 911.0 \pm & 86.51 \pm \\ 0.9204 & 39.1 & 15.84 \\ & 1166.9 \pm & 111.24 \pm \\ 0.9215 & 49.2 & 17.03 \\ & 1462.4 \pm & 139.72 \pm \\ 0.9215 & 62.2 & 18.49 \\ & & \end{array}$

$\begin{array}{llll} & 551.9 \pm & 53.67 \pm & \\ 0.9898 & 17.9 & 13.27 & 0.9776 \\ & 411.3 \pm & 39.65 \pm & \\ 0.9792 & 13.4 & 12.56 & 0.9770 \\ & 267.2 \pm & 25.47 \pm & \\ 0.9782 & 9.0 & 11.74 & 0.9758 \\ & 195.1 \pm & 18.28 \pm & \\ 0.9771 & 6.7 & 11.24 & 0.9745 \\ & 644.4 \pm & 58.79 \pm & \\ 0.9779 & 29.4 & 14.85 & 0.9555 \\ & 739.4 \pm & 68.28 \pm & \\ 0.9792 & 29.5 & 14.86 & 0.9669 \\ & 773.2 \pm & 71.56 \pm & \\ 0.9796 & 29.5 & 14.87 & 0.9699 \\ & 1310.0 \pm & 121.30 \pm & \\ 0.9785 & 52.2 & 17.57 & 0.9637 \\ & 1430.9 \pm & 131.41 \pm & \\ 0.9793 & 59.0 & 18.33 & 0.9641 \\ & 1567.6 \pm & 144.79 \pm & \\ 0.9801 & 59.1 & 18.34 & 0.9707 \\ & 1476.3 \pm & 134.84 \pm & \\ 0.9796 & 59.0 & 18.33 & 0.9666 \\ & 843.9 \pm & 81.60 \pm & \\ 0.9801 & 26.9 & 14.52 & 0.9781 \\ & 1081.4 \pm & 107.60 \pm & \\ 0.9808 & 34.4 & 15.47 & 0.9810 \\ & 1355.7 \pm & 139.77 \pm & \\ 0.9805 & 46.4 & 16.90 & 0.9794\end{array}$

(b) Differential

\begin{tabular}{|c|c|c|}
\hline $\mathrm{A} 3 / 2$ & $\begin{array}{l}520.5 \pm \\
38.4\end{array}$ & $\begin{array}{l}45.15 \pm \\
3.70\end{array}$ \\
\hline A2 & $\begin{array}{l}362.7 \pm \\
42.0\end{array}$ & $\begin{array}{l}29.75 \pm \\
4.05\end{array}$ \\
\hline A3 & $\begin{array}{l}205.8 \pm \\
28.0\end{array}$ & $\begin{array}{l}14.28 \pm \\
2.70\end{array}$ \\
\hline A4 & $\begin{array}{l}143.2 \pm \\
28.5\end{array}$ & $\begin{array}{l}8.00 \pm \\
2.74\end{array}$ \\
\hline R1 & $\begin{array}{l}161.1 \pm \\
38.8\end{array}$ & $\begin{array}{l}10.09 \pm \\
3.74\end{array}$ \\
\hline $\mathrm{R} 2$ & $\begin{array}{l}562.1 \pm \\
45.1\end{array}$ & $\begin{array}{l}48.35 \pm \\
4.34\end{array}$ \\
\hline R3 & $\begin{array}{l}653.5 \pm \\
45.1\end{array}$ & $\begin{array}{l}56.86 \pm \\
4.34\end{array}$ \\
\hline D1 & $\begin{array}{l}992.1 \pm \\
91.0\end{array}$ & $\begin{array}{l}89.97 \pm \\
8.77\end{array}$ \\
\hline D2 & $\begin{array}{l}1235.1 \\
\pm 103.0\end{array}$ & $\begin{array}{l}113.01 \pm \\
9.93\end{array}$ \\
\hline D3 & $\begin{array}{l}1512.7 \\
\pm 123.3\end{array}$ & $\begin{array}{l}138.58 \pm \\
11.88\end{array}$ \\
\hline D4 & $\begin{array}{l}1330.0 \\
\pm 109.1\end{array}$ & $\begin{array}{l}120.76 \pm \\
10.52\end{array}$ \\
\hline $\mathrm{F} 1$ & $\begin{array}{l}836.2 \pm \\
52.8\end{array}$ & $\begin{array}{l}75.79 \pm \\
5.09\end{array}$ \\
\hline $\mathrm{F} 2$ & $\begin{array}{l}1384.4 \\
\pm 105.2\end{array}$ & $\begin{array}{l}129.27 \pm \\
10.14\end{array}$ \\
\hline F3 & $\begin{array}{l}1932.6 \\
\pm 168.4\end{array}$ & $\begin{array}{l}182.76 \pm \\
16.24\end{array}$ \\
\hline
\end{tabular}

$\begin{array}{llllll} & 455.8 \pm & 39.11 \pm & & 495.6 \pm & 42.99 \pm \\ 0.9150 & 7.6 & 0.72 & 0.9937 & 23.7 & 2.25 \\ & 280.1 \pm & 22.14 \pm & & 328.5 \pm & 26.94 \pm \\ 0.8123 & 6.7 & 0.64 & 0.9869 & 26.3 & 2.50 \\ & 104.6 \pm & 5.07 \pm & & 161.4 \pm & 10.76 \pm \\ 0.8277 & 6.7 & 0.64 & 0.9136 & 29.6 & 2.81 \\ & 16.9 \pm & -3.55 \pm & & 77.9 \pm & 2.59 \pm \\ 0.6881 & 7.0 & 0.67 & 0.1731 & 31.4 & 2.99 \\ & 290.8 \pm & 22.94 \pm & & 251.5 \pm & 19.37 \pm \\ 0.5038 & 32.0 & 3.06 & 0.8110 & 43.1 & 4.09 \\ & 502.1 \pm & 42.73 \pm & & 540.7 \pm & 46.46 \pm \\ 0.9010 & 21.3 & 2.03 & 0.9603 & 30.5 & 2.90 \\ & 603.7 \pm & 52.13 \pm & & 637.1 \pm & 55.32 \pm \\ 0.9249 & 17.5 & 1.67 & 0.9810 & 26.8 & 2.55 \\ & 980.1 \pm & 88.56 \pm & & 997.6 \pm & 90.01 \pm \\ 0.8740 & 55.2 & 5.27 & 0.9318 & 56.0 & 5.31 \\ & 1250.7 \pm & 114.01 \pm & & 1254.1 \pm & 113.98 \pm \\ 0.8936 & 46.2 & 4.42 & 0.9695 & 47.9 & 4.55 \\ & 1559.3 \pm & 142.31 \pm & & 1547.0 \pm & 140.61 \pm \\ 0.8980 & 34.4 & 3.29 & 0.9889 & 39.9 & 3.79 \\ & 1356.1 \pm & 122.69 \pm & & 1354.2 \pm & 122.09 \pm \\ 0.8967 & 42.0 & 4.02 & 0.9784 & 44.7 & 4.24 \\ & 806.6 \pm & 72.83 \pm & & 829.9 \pm & 74.93 \pm \\ 0.9363 & 10.8 & 1.03 & 0.9959 & 21.2 & 2.01 \\ & 1416.3 \pm & 131.72 \pm & & 1408.2 \pm & 130.49 \pm \\ 0.9101 & 18.6 & 1.77 & 0.9961 & 28.9 & 2.74 \\ & 2025.8 \pm & 190.58 \pm & & 1986.6 \pm & 186.05 \pm \\ 0.8849 & 42.1 & 4.02 & 0.9901 & 54.8 & 5.20 \\ & & & & & \end{array}$

\begin{tabular}{|c|c|c|}
\hline 0.9500 & $\begin{array}{l}420.9 \pm \\
8.6\end{array}$ & $\begin{array}{l}35.88 \pm \\
0.81\end{array}$ \\
\hline 0.8706 & $\begin{array}{l}266.2 \pm \\
8.4\end{array}$ & $\begin{array}{l}21.08 \pm \\
0.79\end{array}$ \\
\hline 0.5551 & $\begin{array}{l}111.5 \pm \\
8.9\end{array}$ & $\begin{array}{l}6.16 \pm \\
0.85\end{array}$ \\
\hline 0.1824 & $\begin{array}{l}34.2 \pm \\
9.5\end{array}$ & $\begin{array}{l}-1.39 \pm \\
0.90\end{array}$ \\
\hline 0.5895 & $\begin{array}{l}194.1 \pm \\
31.7\end{array}$ & $\begin{array}{l}14.02 \pm \\
3.00\end{array}$ \\
\hline 0.9316 & $\begin{array}{l}462.2 \pm \\
20.7\end{array}$ & $\begin{array}{l}38.98 \pm \\
1.96\end{array}$ \\
\hline 0.9607 & $\begin{array}{l}551.6 \pm \\
17.3\end{array}$ & $\begin{array}{l}47.13 \pm \\
1.63\end{array}$ \\
\hline 0.9323 & $\begin{array}{l}884.5 \pm \\
50.1\end{array}$ & $\begin{array}{l}79.05 \pm \\
4.73\end{array}$ \\
\hline 0.9674 & $\begin{array}{l}1122.3 \pm \\
42.2\end{array}$ & $\begin{array}{l}101.13 \pm \\
3.99\end{array}$ \\
\hline 0.9849 & $\begin{array}{l}1393.8 \pm \\
32.5\end{array}$ & $\begin{array}{l}125.60 \pm \\
3.07\end{array}$ \\
\hline 0.9756 & $\begin{array}{l}1215.0 \pm \\
38.7\end{array}$ & $\begin{array}{l}108.50 \pm \\
3.65\end{array}$ \\
\hline 0.9852 & $\begin{array}{l}730.3 \pm \\
11.3\end{array}$ & $\begin{array}{l}65.33 \pm \\
1.07\end{array}$ \\
\hline 0.9904 & $\begin{array}{l}1266.4 \pm \\
18.5\end{array}$ & $\begin{array}{l}116.63 \pm \\
1.74\end{array}$ \\
\hline 0.9828 & $\begin{array}{l}1802.6 \pm \\
40.6\end{array}$ & $\begin{array}{l}167.93 \pm \\
3.83\end{array}$ \\
\hline
\end{tabular}

$\begin{array}{lll} & 371.2 \pm & 31.21 \pm \\ 0.9905 & 25.7 & 2.42 \\ & 224.1 \pm & 17.18 \pm \\ 0.9778 & 23.1 & 2.17 \\ & 77.0 \pm & 3.03 \pm \\ 0.8703 & 21.1 & 1.98 \\ & 3.4 \pm & -4.13 \pm \\ 0.3405 & 20.4 & 1.92 \\ & 154.9 \pm & 10.43 \pm \\ 0.6133 & 42.0 & 3.95 \\ & 410.2 \pm & 34.07 \pm \\ 0.9557 & 35.2 & 3.32 \\ & 495.2 \pm & 41.78 \pm \\ 0.9779 & 33.7 & 3.18 \\ & 811.2 \pm & 71.98 \pm \\ 0.9311 & 63.7 & 5.99 \\ & 1037.6 \pm & 92.89 \pm \\ 0.9684 & 60.0 & 5.64 \\ & 1296.1 \pm & 116.03 \pm \\ 0.9876 & 56.5 & 5.32 \\ & 1125.9 \pm & 99.81 \pm \\ 0.9772 & 58.4 & 5.49 \\ & 665.4 \pm & 59.10 \pm \\ 0.9946 & 32.2 & 3.03 \\ & 1175.9 \pm & 107.78 \pm \\ 0.9951 & 39.8 & 3.74 \\ & 1686.3 \pm & 156.45 \pm \\ 0.9885 & 58.3 & 5.48 \\ & & \end{array}$

0.9001

0.8021

0.3491

$-0.0441$

0.3546

0.8540

0.9031

0.8751

0.9285

0.9581

0.9416

0.9487

0.9743

0.9732 
J.M. Pérez, J.Ma. Rincón, M. Romero, Study of mullite formation in porcelain stoneware applying isoconversional and IKP methods. Ceramics International, Volume 36, Issue 8, December 2010, Pages 2329-2335; doi:10.1016/j.ceramint.2010.08.001.

As can be seen in Table 2, the integral method gives, in general, higher activation energies than the differential method [8]. The parameters obtained at a heating rate of $10 \mathrm{~K} \mathrm{~min}^{-1}$ have the worst $r$-squared fit in both differential and integral methods. This is because at such low heating rates, the shape of the thermogram is less defined [3] than at higher heating rates, so it is expected that this heating rate brings out more error. The rest of the heating rates have a better fit to the experimental data. A high $r$-squared fit is the first requirement but not the only one. A consistent pre-exponential factor and activation energy values are mandatory.

For the integral method (Table 2a), $r$-squared values are higher (taking into consideration most of the heating rates) for $F n$ and $A_{3 / 2}$, but $F n$ has a higher activation energy than $A_{3 / 2}$. For the differential method, the $r$-squared value is also higher for $F n$ and $A_{3 / 2}$ but the activation energy, which is quite similar to a previous paper [3], is obtained by $R_{3}$, in which the $r$-squared value is lower than the others just mentioned. Using $R_{3}$ from differential ( Table $2 \mathrm{~b}$ ), it can be checked that this Ea value is close to that obtained in the previous paper, where the Ligero et al. method is applied.

Table 2 shows that many models result in clear regression and that it is therefore not possible to decide which is the best. So, by employing just one heating rate, there is no way to take a suitable $f(\alpha)$. This table just gives us the chance to rule out some models ( $D n, R_{1}$ and $\left.R_{2}\right)$.

Currently, we can state that activation energy is over $600 \mathrm{~kJ} \mathrm{~mol}^{-1}$ from all the models assayed.

\subsection{IKR method}

It can be also observed in Fig. 1 that, in the range $0.2-0.6$, the activation energy does not depend on the crystallisation degree. This range is where we are going to focus the application of the IKP method.

Table 3 gives the results of applying the compensation effect to the conversion of models in the integral data. Differential results are not shown because they do not fit well. The results here presented are very well fitted and the $T_{\text {iso }}$ obtained are in range with the crystallisation peak [3]. The $T_{\text {iso }}$ obtained with $A_{3 / 2}$ or $F n$ models are close to the previous work $\left(\approx 985^{\circ} \mathrm{C}\right)$. So, after applying this criterion, it is not possible to take an appropriate model, as was pointed out in Section 2.2.3. 
J.M. Pérez, J.Ma. Rincón, M. Romero, Study of mullite formation in porcelain stoneware applying isoconversional and IKP methods. Ceramics International, Volume 36, Issue 8, December 2010, Pages 2329-2335; doi:10.1016/j.ceramint.2010.08.001.

Table 3. Compensation effect in relation to conversion (Eq. (8) from Table 2a).

\begin{tabular}{ccccc} 
Model & $\alpha^{*}\left(\mathrm{~min}^{-1}\right)$ & $\beta^{*}\left(\mathrm{~mol} \mathrm{~kJ}{ }^{-1}\right)$ & Tiso $\left({ }^{\circ} \mathrm{C}\right)$ & $\mathrm{r}^{2}$ \\
\hline A3/2 & $-1.3361 \pm 0.0450$ & $0.0969 \pm 0.0001$ & 967.6 & 10.000 \\
A2 & $-3.1147 \pm 0.0577$ & $0.0998 \pm 0.0001$ & 932.4 & 10.000 \\
A3 & $-2.0996 \pm 0.1633$ & $0.0984 \pm 0.0003$ & 949.6 & 0.9999 \\
A4 & $-5.7826 \pm 0.1871$ & $0.1040 \pm 0.0003$ & 883.3 & 10.000 \\
R1 & $-0.9891 \pm 0.3977$ & $0.0957 \pm 0.0006$ & 984.1 & 0.9998 \\
R2 & $-0.1752 \pm 0.3218$ & $0.0936 \pm 0.0005$ & 1011.7 & 0.9999 \\
R3 & $-0.0350 \pm 0.2899$ & $0.0929 \pm 0.0004$ & 1022.0 & 0.9999 \\
D1 & $6.4723 \pm 1.1006$ & $0.0831 \pm 0.0017$ & 1174.5 & 0.9980 \\
D2 & $7.6712 \pm 1.0205$ & $0.0805 \pm 0.0015$ & 1220.7 & 0.9982 \\
D3 & $8.3805 \pm 0.8921$ & $0.0775 \pm 0.0013$ & 1279.1 & 0.9985 \\
D4 & $-9.4420 \pm 0.4947$ & $0.1094 \pm 0.0007$ & 825.9 & 0.9998 \\
F1 & $2.2212 \pm 0.2168$ & $0.0913 \pm 0.0003$ & 1044.5 & 0.9999 \\
F2 & $-1.2389 \pm 0.8050$ & $0.0984 \pm 0.0012$ & 949.7 & 0.9992 \\
F3 & $5.9726 \pm 1.3118$ & $0.0885 \pm 0.0020$ & 1086.5 & 0.9975
\end{tabular}

The compensation effect is also applied to the data (heating rate) in Table 2 (except those data showing worse fit), and the results are listed in Table 4 . The correlated data are very similar to those shown in Table 3 . The $T_{\text {iso }}$ is also in the experimental range and in agreement with Table 3. In Table 4, $r$-squared data are slightly better in the differential data than in the integral data because, to calculate $T_{\text {iso }}$ by differential models, we have selected the best-fit $f(x)$ functions. That is why there are less differential models than integral models, so the error of fitting in the compensation effect is lower.

Table 4. Values of integral and differential compensation parameters for data from Table $2 \mathrm{a}$ and $\mathrm{b}$ for all heating rates.

\begin{tabular}{|c|c|c|c|c|c|c|c|c|}
\hline$\beta\left(\mathrm{K} \min ^{-1}\right)$ & $\begin{array}{c}\text { Integral } \\
\mathrm{a}^{*}\left(\min ^{-1}\right)\end{array}$ & $\begin{array}{l}\text { Differential } \\
\mathrm{b}^{*}\left(\mathrm{~mol} \mathrm{~kJ}^{-1}\right)\end{array}$ & $\mathrm{T}_{\text {iso }}\left({ }^{\circ} \mathrm{C}\right)$ & $r^{2}$ & $a^{*}\left(\min ^{-1}\right)$ & $\mathrm{b}^{*}\left(\mathrm{~mol} \mathrm{~kJ}{ }^{-1}\right)$ & $\mathrm{T}_{\text {iso }}\left({ }^{\circ} \mathrm{C}\right)$ & $\mathrm{r}^{2}$ \\
\hline 10 & $\begin{array}{c}-1.5151 \pm \\
0.9039\end{array}$ & $\begin{array}{c}0.0966 \pm \\
0.0009\end{array}$ & 972.5 & 0.9993 & $\begin{array}{c}-5.6993 \pm \\
0.5326\end{array}$ & $\begin{array}{c}0.0966 \pm \\
0.0005\end{array}$ & 972.6 & 0.9996 \\
\hline 20 & $\begin{array}{c}-0.8106 \pm \\
0.6618\end{array}$ & $\begin{array}{c}0.0956 \pm \\
0.0006\end{array}$ & 985.2 & 0.9996 & $\begin{array}{c}-5.0798 \pm \\
0.4988\end{array}$ & $\begin{array}{c}0.0957 \pm \\
0.0005\end{array}$ & 984.1 & 0.9997 \\
\hline 30 & $\begin{array}{c}-0.1316 \pm \\
0.5960\end{array}$ & $\begin{array}{c}0.0945 \pm \\
0.0005\end{array}$ & 999.1 & 0.9996 & $\begin{array}{c}-4.7244 \pm \\
0.5188\end{array}$ & $\begin{array}{c}0.0951 \pm \\
0.0005\end{array}$ & 991.7 & 0.9996 \\
\hline 40 & $\begin{array}{c}0.0991 \pm \\
0.5930\end{array}$ & $\begin{array}{c}0.0941 \pm \\
0.0005\end{array}$ & 1005.4 & 0.9996 & $\begin{array}{c}-4.5359 \pm \\
0.5013\end{array}$ & $\begin{array}{c}0.0946 \pm \\
0.0005\end{array}$ & 997.7 & 0.9996 \\
\hline 50 & $\begin{array}{c}0.3271 \pm \\
0.9406\end{array}$ & $\begin{array}{c}0.0936 \pm \\
0.0009\end{array}$ & 1011.8 & 0.9991 & $\begin{array}{c}-4.3651 \pm \\
0.4875\end{array}$ & $\begin{array}{c}0.0943 \pm \\
0.0006\end{array}$ & 1002.8 & 0.9995 \\
\hline
\end{tabular}


J.M. Pérez, J.Ma. Rincón, M. Romero, Study of mullite formation in porcelain stoneware applying isoconversional and IKP methods. Ceramics International, Volume 36, Issue 8, December 2010, Pages 2329-2335; doi:10.1016/j.ceramint.2010.08.001.

\subsection{IKP method}

The invariant kinetic parameters obtained are listed in Table 5 using differential and integral compensation parameters from Table 4 (Eq. (9)).

Table 5. Invariant parameters.

\begin{tabular}{ccc} 
& Integral & Differential \\
\hline $\mathrm{Ea}_{\text {inv }}\left(\mathrm{kJ} \mathrm{mol}^{-1}\right)$ & $628.2 \pm 26.5$ & $584.1 \pm 36.9$ \\
$\mathrm{Ln} \mathrm{A}_{\text {inv }}\left(\mathrm{min}^{-1}\right)$ & $59.19 \pm 2.51$ & $50.75 \pm 3.52$ \\
$\mathrm{r}^{2}$ & 0.9929 & 0.9842
\end{tabular}

The Ea and $\operatorname{Ln} A$ values obtained are very similar to those given in the previous paper [3]. The data are similar to model $A_{3 / 2}$ under the integral method and $R_{2}-R_{3}$ under the differential method ( Table 2). To test which model is correct, the Pérez-Maqueda et al. criterion has been employed (see below).

As can be seen, the invariant activation energies obtained by integral and differential models are similar. Both are close to the activation energies obtained in previous work.

\subsection{Pérez-Maqueda method}

The $\operatorname{Ln}\left[\beta g(x) / T^{2}\right] v s .1 / T\left(\mathrm{~K}^{-1}\right)$ curves for all heating rates and $g(\alpha)$ were drawn. According to the Pérez-Maqueda et al. criterion, the correct kinetic model corresponds to the independence of the activation parameters on the heating rate. In Fig. 2, all the points lay on the same line for the Johnson-Melh-Avrami model with $n=1.5$. Applying the Pérez-Maqueda et al. criterion, the slope and intercept obtained are $621.2 \mathrm{~kJ} \mathrm{~mol}^{-1}$ and $58.92 \mathrm{~min}^{-1}$ to $\mathrm{Ln} A$. These values are very close to those found previously. 
J.M. Pérez, J.Ma. Rincón, M. Romero, Study of mullite formation in porcelain stoneware applying isoconversional and IKP methods. Ceramics International, Volume 36, Issue 8, December 2010, Pages 2329-2335; doi:10.1016/j.ceramint.2010.08.001.

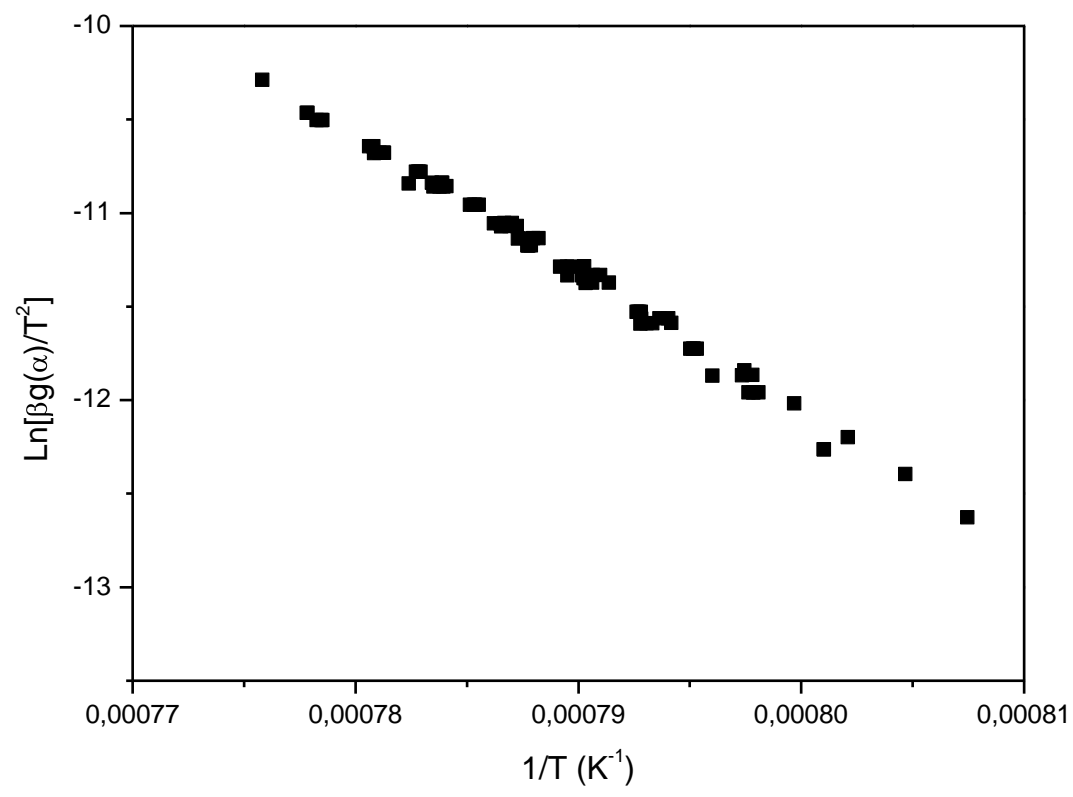

Fig. 2. $\operatorname{Ln}\left[\beta \mathrm{g}(\mathrm{x}) / \mathrm{T}^{2}\right]$ vs. $1 / \mathrm{T}$ by the Johnson-Melh-Avrami method, with $\mathrm{n}=1.5$ for all the heating rates.

The value of $n=1.5$ can be explained by assuming either of the two following mechanisms[29]: (1) instantaneous nucleation (saturation of the sites capable of nucleation prior to the process) and subsequent three-dimensional growth of the nuclei by a diffusion controlled rate, or (2) constant rate of homogeneous nucleation and one-dimensional growth of the nuclei (branching) through a diffusion controlled process. In our case, we can choose the first assumption, as it was probed in a previous paper [3].

\section{Conclusions}

A non-isothermal kinetic study of mullite formation in porcelain stoneware has been performed. Five heating rates have been employed to obtain the activation energy, preexponential factor and, when the method allows, the function of conversion. They have been used an integral and differential single $x=x(T)$ method and two isoconversional methods (Kissinger-Akahira-Sunose and Flynn-Wall-Ozawa). The Isokinetic Relationship was applied to obtain $T_{\text {iso. }}$ The Isokinetic Parameters method was used to obtain both the activation energy 
J.M. Pérez, J.Ma. Rincón, M. Romero, Study of mullite formation in porcelain stoneware applying isoconversional and IKP methods. Ceramics International, Volume 36, Issue 8, December 2010, Pages 2329-2335; doi:10.1016/j.ceramint.2010.08.001.

and the pre-exponential factor invariant. To check the best $f(x)$, the Pérez-Maqueda et al. criterion was applied. As the presented results show, it can be concluded that:

- The activation energy obtained from isoconversional methods is in the range of 600$700 \mathrm{~kJ} \mathrm{~mol}^{-1}$.

- From 0.2 to 0.6 crystallisation conversion, the isoconversional methods are proportionate to the activation energy and do not depend on the degree of crystallisation.

- Integral and differential methods with a single-heating rate do not allow us to choose the proper $f(x)$ for mullite crystallisation.

- The $T_{\text {iso }}$ obtained with differential and integral data is over $985^{\circ} \mathrm{C}$. The isokinetic relationship applied to conversion and heating rates shows similar $T_{\text {iso. }}$.

- The invariant kinetic parameters method was applied to both integral and differential methods. They give similar values for activation energy and pre-exponential factor: $628.2 \mathrm{~kJ} \mathrm{~mol}^{-1}$ and 59.19 , and $584.1 \mathrm{~kJ} \mathrm{~mol}^{-1}$ and 50.75 , respectively.

- The Pérez-Maqueda et al. criterion fits a Johnson-Melh-Avrami model with $n$ equal to 1.5. It gives $\mathrm{Ea}=621.2 \mathrm{~kJ} \mathrm{~mol}^{-1}$ and $\operatorname{Ln} A=58.92$, which is close to the integral data given by $A_{3 / 2}$.

\section{References}

[1] Y. Iqbal, W.E. Lee, Fired porcelain microstructure revisited, J. Am. Ceram. Soc., 82 (12) (1999), pp. 3584-3590.

[2] J.Ma. Rincón, G. Thomas, J.S. Moya, Microstructural study of sintered mullite obtained from premullite, J. Am. Ceram. Soc., 69 (2) (1986), pp. C29-C31.

[3] M. Romero, J. Martín-Márquez, J.Ma. Rincón, Kinetic of mullite formation from a porcelain stoneware body for tiles production, J. Eur. Ceram. Soc., 26 (2006), pp. 1647-1652.

[4] M. Dondi, G. Guarini, C. Melandri, M. Raimondo, P.M.T. Cavalante, C. Zanelli, Resistance to deep abrasion of porcelain stoneware tiles, Ind. Ceram., 25 (2) (2005), pp. 71-78.

[5] C. Leonelli, F. Bondioli, P. Veronesi, M. Romagnoli, T. Manfredini, G.C. Pellacani, V. Cannillo, Enhancing the mechanical properties of porcelain stoneware tiles: a microstructural approach, J. Eur. Ceram. Soc., 21 (2001), pp. 785-793.

[6] E. Sánchez, M.J. Orts, J. García-Tena, V. Cantavella, Porcelain tile composition effect on phase formation and end products, Am. Ceram. Soc. Bull., 80 (6) (2001), pp. 43-49. 
J.M. Pérez, J.Ma. Rincón, M. Romero, Study of mullite formation in porcelain stoneware applying isoconversional and IKP methods. Ceramics International, Volume 36, Issue 8, December 2010, Pages 2329-2335; doi:10.1016/j.ceramint.2010.08.001.

[7] A. Rotaru, A. Moanta, I. Sălăgeanu, P. Budrugeac, E. Segal, Thermal decomposition kinetics of some aromatic azomonoethers. Part I. Decomposition of 4-[(4-chlorobenxyl)oxy]-4'nitro-azobenzene, J. Therm. Anal. Calorim., 87 (2) (2007), pp. 395-400

[8] A. Cadenato, J.M. Morancho, X. Fernández-Francos, J.M. Salla, X. Ramis, Comparative kinetic study of the non-isothermal thermal curing of bis-GMA/TEGDMA systems, J. Them. Anal. Calorim., 89 (1) (2007), pp. 233-244

[9] X. Ramis, J.M. Salla, J. Puiggalí, Kinetic Studies on the thermal polymerization of NChloroacetyl-11-aminoundecanoate potassium salt, J. Polym. Sci. A, 43 (2005), pp. 1166-1176. [10] B. Janković, B. Adnadevic, S. Mentus, The kinetic analysis of non-isothermal nickel oxide reduction in hydrogen atmosphere using the invariant kinetic parameters method, Thermochim. Acta, 456 (2007), pp. 48-55.

[11] O.C. Mocioiu, M. Zaharescu, G. Jitianu, P. Budrugeac, Kinetic parameters determination in non-isothermal conditions for the crystallization of a silica-soda-lead glass, J. Therm. Anal. Calorim., 86 (2) (2006), pp. 429-436.

[12] L.A. Pérez-Maqueda, J.M. Criado, F.G. Gotor, J. Malek, Advantages of combined kinetic analysis of experimental data obtained under any heating profile, J. Phys. Chem., 106 (2002), pp. 2862-2868.

[13] T. Ozawa, A new method of analyzing thermogravimetric data, Bull. Chem. Soc. Jpn., 38 (1) (1965), pp. 1881-1886.

[14] J.H. Flynn, L.A. Wall, General treatment of the thermogravimetry of polymers, J. Res. Natl. Bur. Stand. A: Phys. Chem., 70 (6) (1966), pp. 487-523.

[15] T. Akahira, T. Sunose, Res. Report. Chiba Inst. Technol., 16 (1971), pp. 22-31.

[16] C.D. Doyle, Estimating thermal stability of experimental polymers by empirical thermogravimetric analysis, Anal. Chem., 33 (1961), pp. 77-79

[17] P. Murray, J. White, Kinetics of the thermal de-hydration of clays, Trans. Br. Ceram. Soc., 54 (1955), pp. 151-187.

[18] A.W. Coats, J.P. Redfern, Kinetics parameters from thermogravimetric data, Nature, 201 (1964), pp. 68-69.

[19] S. Vyazovkin, W. Linert, False isokinetic relationships found in the nonisothermal decomposition of solids, Chem. Phys., 193 (1995), pp. 109-118

[20] S. Vyazovkin, W. Linert, The application of isoconversional methods for analyzing isokinetic relationships occurring at thermal decomposition of solids, J. Solid State Chem., 114 (1995), pp. 392-398.

[21] A.I. Lesnikovich, S.V. Levchik, A method of finding invariant values of kinetic parameters, J. Therm. Anal. Calorim., 27 (1983), pp. 89-94. 
J.M. Pérez, J.Ma. Rincón, M. Romero, Study of mullite formation in porcelain stoneware applying isoconversional and IKP methods. Ceramics International, Volume 36, Issue 8, December 2010, Pages 2329-2335; doi:10.1016/j.ceramint.2010.08.001.

[22] A.I. Lesnikovich, S.V. Levchik, Isoparametric kinetic relationship for chemical transformations in condensed substances (analytical survey). II. Reactions involving the participation of solids substances, J. Therm. Anal. Calorim., 30 (1985), pp. 667-702. [23] P. Budrugeac, Some methodological problems concerning the kinetic analysis of nonisothermal data for thermal and thermo-oxidative degradation of polymers and polymeric materials, Polym. Degrad. Stab., 89 (2005), pp. 265-273.

[24] P. Budrugeac, E. Segal, On the kinetic processing of thermal analysis data, Rev. Roum. Chim., 49 (2004), pp. 193-198.

[25] R.A. Ligero, J. Vazquez, M. Casa-Ruiz, R. Jiménez-Garay, A study of the crystallization kinetics of some $\mathrm{Cu}-\mathrm{As}-\mathrm{Te}$ glasses, J. Mater. Sci., 26 (1991), pp. 211-215.

[26] C.R. Li, T.B. Tang, Isoconversion method for kinetic analysis of solid-state reactions from dynamic thermoanalytical data, J. Mater. Sci., 34 (1999), pp. 3467-3470.

[27] T.B. Tang, M.M. Chaudri, Analysis of dynamic kinetic data from solid-state reactions, J. Therm. Anal. Calorim., 18 (1980), pp. 247-261.

[28] C.Y. Chen, G.S. Lan, W.H. Tuan, Microstructural evolution of mullite during the sintering of kaolin powder compacts, Ceram. Int., 26 (2000), pp. 715-720.

[29] J. Šesták, Themophysical Properties of Solids, Elsevier, Amsterdam (1984). 